ISSN 1112-9867

Available online at http://www.jfas.info

\title{
GEOMETRY OF WAVES GUIDES FOR CONTAINMENT OF PROPAGATING RESONANT MODES
}

\author{
Z. Aboura* and B. Bouhafs*
}

Laboratoire de Physique Théorique, Faculté des Sciences, Université Abou-Bekr Belkaid, 13000 Tlemcen

Received: 17 February 2011 / Accepted: 29 April 2010 / Published online: 30 June 2011

\begin{abstract}
We are study de mode fundamental de 1D in the two media (MIM and IMI) of a metallic structure (Ag) (silver) in which a small hole is perforated (as à dielectric medium). We take the conditions that resonant modes (PPSs) are confined or the surface.Via the complex permittivity of the metal and those of the dielectric medium .The effective index $n_{\text {eff }}$ of the elaborated structure controlled remarkably the properties of the propagating modes. Of the structure playing he mode to guide SPP modes .Where the confinement follow the order in sub-wave length scale of the width hole in silver and limited by the attenuation length (skin depth).
\end{abstract}

Key words: metallic structure, effective index, guided SPP modes, skin depth.

\section{INTRODUCTION}

Au cours de ces dix dernières années, le développement des nanotechnologies a permis l'émergence de nouveaux matériaux pour la nano-photonique, tels que les cristaux photoniques, les nanostructures métalliques ou plus précisément les méta-matériaux .La réalisation de ces composants repose sur une structuration à l'échelle sub-longueur d'onde de matériaux diélectriques ou métalliques [1]. Les nouvelles possibilités offertes par une telle structuration sont le contrôle de la propagation des ondes $E M$ (direction, vitesse de groupe, pertes), et la réalisation par exemple de guide d'onde de très faibles dimensions latérales, où le confinement de la lumière se produit dans des volumes extrêmement faibles [2].

Author Correspondence, e-mail: zakiaaboura@yahoo.fr

ICID: 1020782 
D’une manière générale, les nanostructures permettent une augmentation des interactions lumière-matière, avec de nombreuses applications en photoniques et en optoélectronique dans les domaines de l'émission, de la détection, de l'imagerie ou du guidage optique [3]. L'étude de l'interaction lumière-matière avec les nanostructures métalliques, la plasmonique a connu un vif intérêt. La raison vient de la particularité est plasmons de surface qui permettent la propagation de la lumière le long d'une interface. Ces ondes à caractère évanescent sont couplées aux oscillations des électrons libres du métal. Pour leur sensibilité à leur environnement prés de l'interface [3], les plasmons rendent comptes de toute modification de l'indice optique qui est en fait relative à un changement de leur condition de résonnance sur un film métallique [1]-[2]. En mesurant ce décalage, il est possible de détecter la présence de molécules avoisinants le film métallique. Ainsi, les plasmons constituent des détecteurs très sensibles à l'analyse des surfaces dans différents demain en physique, biologie, chimie

Aux perspectives de tous les atouts offerts par les plasmons, nous nous sommes intéressés à l'étude de la propriété principale de guider la lumière à travers une structure. A ce titre, les travaux T.W. Ebbesen [4], A. Degiron [5] et Z.Aboura [3], focalisés sur la transmission extraordinaire de la lumière à travers un film métallique percé de trous sub-longueur d'onde sont certainement marquants pour avoir bien mené la compréhension des mécanismes mis en jeu.

Dans ce travail, nous avons élaboré une géométrie des guides d'ondes pour le confinement des modes propagatifs résonnants (voir la figure 1). De cette idée nous suggérons la recherche de meilleures performances du guide pour un fort confinement optique de ces modes. La caractéristique principale des dimensions est l'échelle sublongueur d'onde par rapport à la longueur d'onde $\lambda$ de l'onde d'excitation [1]-[3]-[6]. Dans leur forme la plus simple, les guides optiques sont constitués par des milieux matériels disposés en couches. Pour que l'énergie lumineuse puisse être propagée et confinée à travers le guide, les indices de ces milieux sont consignés par les conditions usuelles optiques (absorption et dispersion) [6].

\section{MANIPULATION DES ONDES ELECTROMAGNETIQUES A TRAVERS UNE OUVERTURE NANOMETRIQUE DANS UN METAL (AG)}

Des ouvertures nanométriques percées dans une surface métallique se décrivent par des propriétés optiques conduisant à l'apparition de champ électrique d'une amplitude très importante identifiée par la transmission exaltée [6]. C'est le phénomène de 
confinement des modes plasmons polaritons de la surface (PPS) induit par le couplage optique des photons incidents sur la structure et les électrons libres de conduction localisés en surface du matériau impliqué.

Plusieurs structures ont été suggérées dans la littérature [7]. L'efficacité en transmission à travers une simple ouverture sub-longueur d'onde dans un film métallique peut atteindre l'effet tunnel optique à travers le mode fondamental guidé dans l'ouverture. Le rôle clé de l'ouverture sur le processus de transmission de la lumière a été démontré expérimentalement [8] et théoriquement [9]-[2]. La manipulation d'une telle échelle sub-longueur d'onde à travers les ouvertures dans les métaux reste un challenge pour la fabrication des sondes destinées à la microscopie optique en champ proche.

Nous présentons l'étude d'un guide d'onde métallique à une dimension quỉl s'agit d'un milieu diélectrique de permittivité $\varepsilon_{\mathrm{d}}$ et de largeur w entouré d'un conducteur métallique semi-infini de permittivité $\varepsilon_{\mathrm{m}}$. On note par $\mathrm{k}$ le vecteur d'onde du mode fondamental guidé et $\mathrm{k}_{0}=2 \pi / \lambda$ et le vecteur d'onde de la propagation dans l'espace libre.
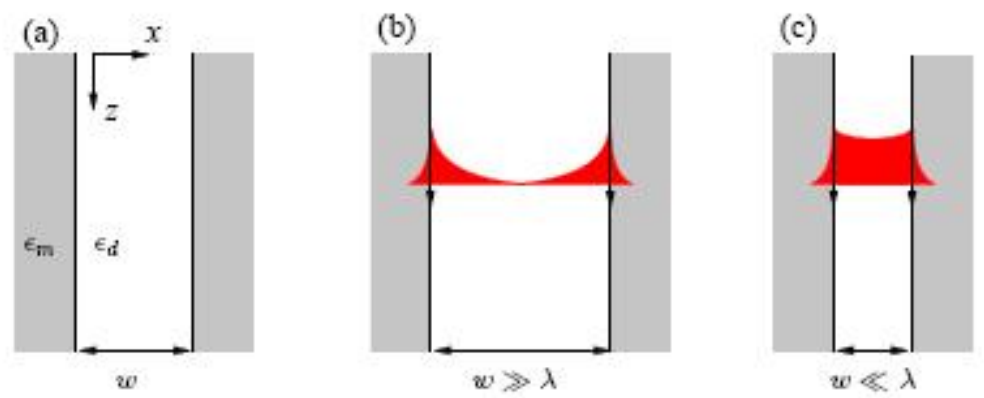

Fig.1. Représentation schématique ((a) : d'un guide d'onde métallique plan à 1D d'un guide d'onde est composé de deux ondes plasmons de surface (en noir); (b): Ces ondes sont faiblement couplées dans les largeurs du guide d'ondes et (c): sont fortement couplées dans le cas de largeurs plus rétrécies).

Dans le cas de parois métalliques parfaites, le mode fondamental du guide d'onde est un mode transfert électromagnétique (TEM) dont le vecteur d'onde axial est $k=\sqrt{\varepsilon_{d}} k_{o}$ ou $k$ est indépendant de la largeur w du guide d'onde, et il est claire qu'il n'ya pas de fréquence de coupure. Dans le cas ou le métal est non parfait, l'équation exacte du mode 1D dans le guide est donnée par [3] : 


$$
* \frac{k_{x . d}}{\varepsilon_{d}}\left[\frac{1-e^{i k x d} w}{1-e^{i k x d} w}\right]+\frac{k_{x . m}}{\varepsilon_{m}}=0
$$

ou $k_{x . d}=\sqrt{\varepsilon_{d} k_{0}^{2}-k^{2}}$ représente vecteur d'onde de la propagation axial de diélectrique, et $k_{x . m}=\sqrt{\varepsilon_{m} k_{0}^{2}-k^{2}}$ vecteur d'onde de la propagation axial du métal.

L'équation (1) se devient sous la forme suivant :

$$
\frac{k_{x . d}}{\boldsymbol{\varepsilon}_{d}}+\frac{k_{x . m}}{\boldsymbol{\varepsilon}_{m}}=0
$$

Cette dernière expression représente [3], la relation exacte de la dispersion des plasmons -polaritons de surface (PPSs) sur interface semi- infinie diélectrique -métal.

L'indice effectif de ce mode est donnée par la formule suivant [3] :

$$
n_{\text {eff }}=\frac{k}{k_{0}}=\frac{1}{\sqrt{\frac{1}{\varepsilon_{d}}+\frac{1}{\varepsilon_{m}}}}
$$

Dans ce type propagation de deux SPPs, on a deux formes de couplages :

1-) Le mode fondamental TM est composé d'une propagation de deux SPPs non couplés le long des parois des deux interfaces diélectrique-métal dans la largeur du guide (Fig. 1b) $\quad w>>\lambda$.

2-) Pour les guides plus rétrécis c'est-à-dire dans une échelle sub longueur d'onde le couplage entre les deux SPPs ne pas être négligé ( Fig 1c) $w<<\lambda$.

Dans ce cas ou le couplage est plus importants c-à-d: $\left|\mathrm{k}_{\mathrm{xd}} \mathrm{W}\right|<<1$, la formule est donnée plus simple sous la forme suivants :

$$
\frac{1-e^{i k x d \cdot w}}{1+e^{i k x d^{\cdot w}}} \approx-\frac{i k_{x \cdot d} w}{2}
$$

En substituant maintenant l'équation (4) dans l'équation (1), on peut écrire comme équation :

$$
\frac{k_{x . m}}{\boldsymbol{\varepsilon}_{m}}=\frac{k_{x \cdot d}}{\varepsilon_{d}}\left(\frac{i k_{x . d} \cdot w}{2}\right)
$$

Avec : 


$$
k_{x . d}^{2}-k_{x . m}^{2}=\left(\varepsilon_{d}-\varepsilon_{m}\right) k_{0}^{2}
$$

En substituant maintenant l'équation (6) dans l'équation (5), on, trouve comme équation

$$
k_{x . d}^{2}\left[1+k_{x . d}^{2}\left(\frac{\boldsymbol{\varepsilon}_{m}}{\boldsymbol{\varepsilon}_{d}}\right)^{2} \frac{w^{2}}{4}\right]=\left(\boldsymbol{\varepsilon}_{d}-\boldsymbol{\varepsilon}_{m}\right) k_{0}^{2}
$$

Ainsi, nous obtenons une expression analytique de l'indice effectif du mode fondamental $\mathrm{TM}_{0}$ décrivant le couplage des SPPs [3] et on trouvons comme équation final :

$$
n_{1 . D}=\frac{k}{k_{0}}=\sqrt{\varepsilon_{d}}\left(1+\frac{\lambda}{\pi w \sqrt{-\varepsilon_{m}}} \sqrt{1+\frac{\varepsilon_{d}}{-\varepsilon_{m}}}\right)^{1 / 2}
$$

Données atomiques

Nous tenons à préciser que nous travaillons dans les conditions d'exciter l'onde plasmon polariton de surface par une onde électromagnétique dans le fait de prendre la partie réelle négative de la permittivité diélectrique du métal. Les paramètres caractéristiques $\mathrm{du}$ guide sont la profondeur de propagation $\delta_{\mathrm{c}}$ de l'onde électromagnétique dans le milieu métallique les permittivités du métal et celle du milieu de l'ouverture, les dimensions (longueur et largeur) du guide, la relation de la dispersion dépend essentiellement l'indice effectif 1D, et la fréquence de coupure.

Pour le calcule numériquement l'indice effectif $\mathrm{n}_{1 \mathrm{D}}$ en fonction de rapport réduit $\mathrm{w}>>$ $\lambda$, en utilisons un programmation Fortran développé, les donnés adopter par la référence $[3]$.

\section{RESULTATS ET DISCUSSIONS}

Dans cette partie, nous reportons dans les figures 2, 3 les résultats numériques l'indice effectif $\mathrm{n}_{1 \mathrm{D}}$ en fonction de rapport réduit $\mathrm{w}>>\lambda$, permettant de décrire les modes de propagation d'une onde électromagnétique dans un guide d'onde dans métal Ag. 


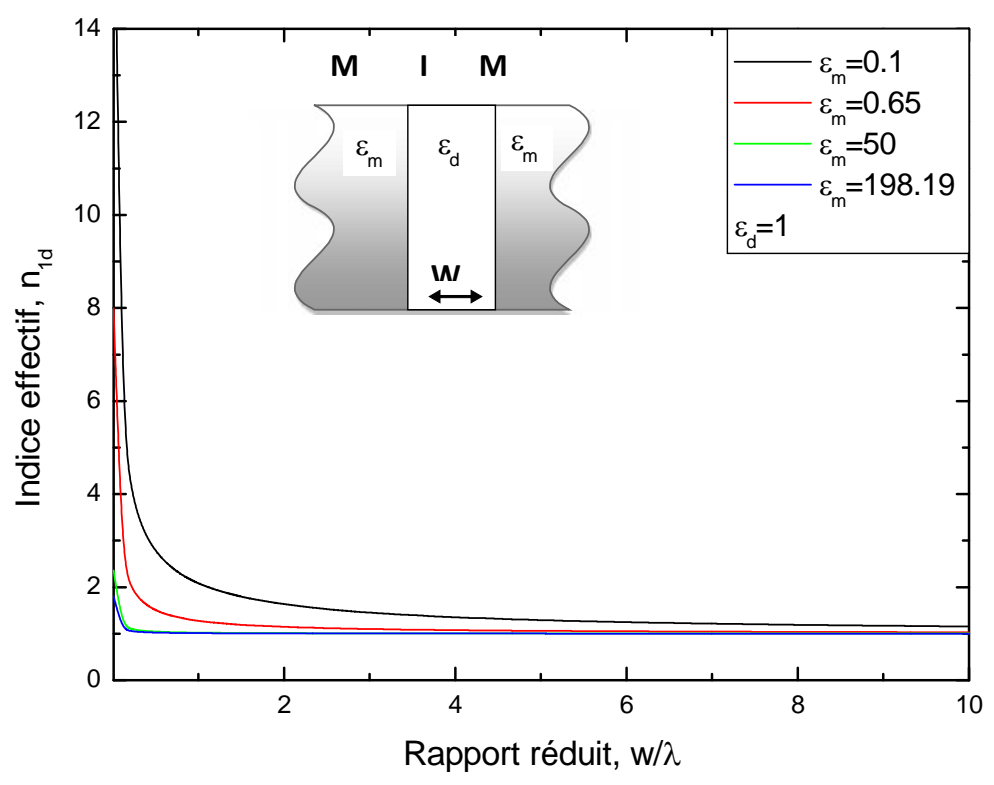

Fig.2. Comportement de l'indice effectif $\mathrm{n}_{1 \mathrm{~d}} \mathrm{du}$ mode fondamental TM d'un guide métallique plan du type Ag-diélectrique-Ag. Le diélectrique est de permittivité $\varepsilon_{\mathrm{d}}=1$.

On représente sur la figure 2 , comportement de l'indice effectif $\mathrm{n}_{1 \mathrm{~d}} \mathrm{du}$ mode fondamental transfert magnétiques (TM) d'un guide métallique plan du type Agdiélectrique-Ag. En se fixant une ouverture de largeur w et en adoptant l'Ag comme milieu métallique de différents valeurs de sa permittivité $\left|\varepsilon_{\mathrm{m}}\right|=0.1,0.65,50$ et 198,19, respectivement. On reporte le profil de $n_{\text {eff }}$ en fonction du rapport réduit $w / \lambda$. La présence d'un faible couplage des plasmons polaritons de surface au seuil $w>0 . \lambda$ dans la condition $\left|\varepsilon_{\mathrm{m}}\right| \geq 50$.

Le fort couplage ainsi induit se traduit par un indice effectif plus important .Par contre pour le cas de grandes valeurs du rapport w/ $\lambda$ l'indice effectif admet la solution d'un métal parfait traduite par la branche asymptotique .Depuis un maximum, ce comportement se décrit par une décroissance tendant vers une limite constante légèrement distingué pour des faibles valeurs du terme $\varepsilon_{\mathrm{m}}$ consignant la dispersion optique de l'Ag pour un rapport $w / \lambda=2.59$

Nous reportons le profil de l'indice effectif en intervertissant cette fois les deux milieux du guide, les conditions sur le milieu externe (diélectrique -Ag- diélectrique) sont indiquées sur la 
Figure 3, avec $\left|\varepsilon_{\mathrm{m}}\right|=198.19$ et $\varepsilon_{\mathrm{d}}=1.5,2.3$ et 3 , respectivement.

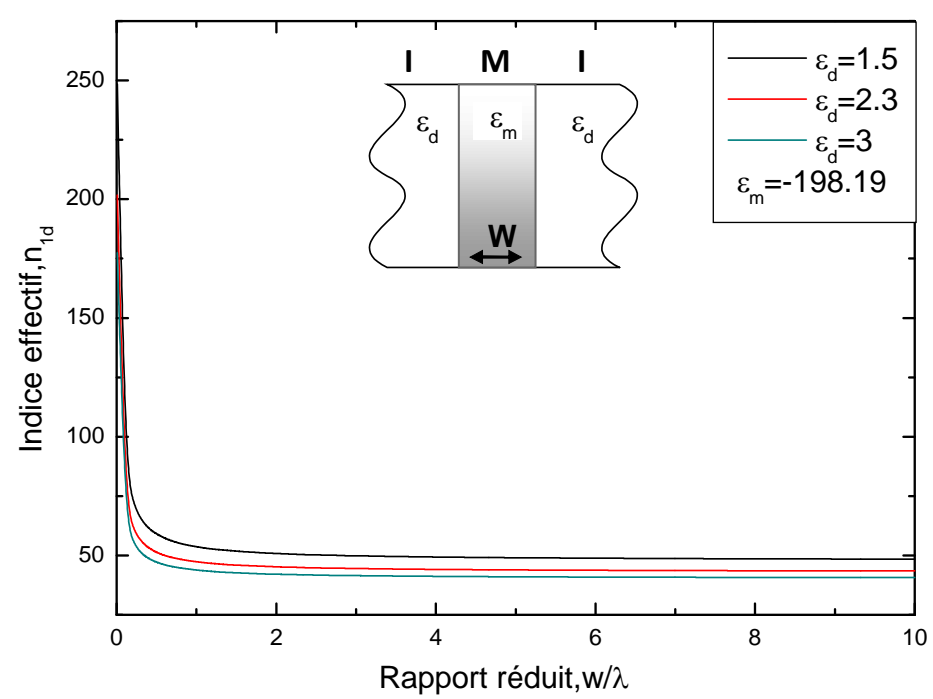

Fig.3. Comportement de l'indice effectif $\mathrm{n}_{1 \mathrm{~d}} \mathrm{du}$ mode fondamental TM d'un guide métallique plan du type diélectrique-Ag-diélectrique. Le diélectrique est de permittivité $\varepsilon_{\mathrm{d}}=1.5,2.3$ et 3 .

Dans cette figure, nous considérons une forte contribution en dispersion sur la permittivité du métal. La valeur du seuil de l'indice effectif sur le rapport $w / \lambda$ est augmentée par la diminution de la permittivité $\varepsilon_{d}$ du milieu externe du guide .Au delà du seuil minimum, la décroissance de l'indice effectif en terme du rapport $w / \lambda$ est légèrement identique où la dimension de l'ouverture est plus grande que la longueur d'onde .La sensibilité de l'indice effectif dans ce cas IMI (figure3) par rapport au cas précédent MIM (figure2) devient plus forte pour tout le rapport $w / \lambda$.

Le milieu externe augmente le couplage des modes plasmon -polaritons de surface (PPS) pour une faible ouverture w devant la longueur d'onde.

\section{CONCLUSION}

Nous avons étudieé analytiquement les propriétés des modes plasmons de surface sur un film d'argent $(\mathrm{Ag})$ percé d'une ouverture, ces modes sont confiné à la surface du matériau et se propagent le long de celle-ci. Ces modes appelés ondes de surface se couplent aux ondes propagations via l'ouverture du guide constitué. Au moyen de la fonction analytique (indice effectif) sur laquelle est basée l'étude de ces modes à 
caractère évanescent nous parvenons à une estimation quantitative des dimensions de l'ouverture à une échelle nanométrique plus faible que la longueur d'onde incidente. Ainsi, il est maîtrisé l'influence des paramètres géométriques et optiques de la structure en vue l'étude de la transmission extraordinaire.

A base des résultats obtenus, nous avons montré que les modes du guide dépendent de sa forme (dimensions) et de l'indice du milieu diélectrique. Cette dépendance offre le moyen de contrôler la langueur de propagation en fonction de la longueur d'onde de la lumière dans le guide plasmonique nano-structuré.

\section{BIBLIOGRAPHIE}

[1] Garcia-Vidal F.J., Martin-Moreno L, et Esteban Moreno L. K. S, Phys. Rev. B 74, 2006, Transmission of light through a single rectangular hole in a real metal,153411.

[2] Garcia-VidalF.J.,Martin-Moreno L., and Pendry J. B., J. Opt. A 7, S97, 2005, Surfaces with holes in them: new plasmonic metamaterials.

[3] Z.Aboura, 2010, Geometries des guides d'ondes pour le confinement des modes propagatifs resonnants., mémoire de Magister, Université de Tlemcen, 4-30 P. [4] EbbesenT.W., Lezec .H. J.,et Ghaemi H. F., 1998 , Exraordinary optical transmission through sub-wavelength hole arrays , 667391. [5] Degiron A., and Ebbesen T.W.,J.Opt .A 7,590,2005, The role of localized surface plasma modes in the enhanced transmission ot periodic sub wavelength apertures,. [6] William L.,et al , 2003 , Surface Plasmon sub wavelength optics , 824, 424. [7] Hervé Rigneaut, Jeremy Capoulade et al , Phys. Rev .Lett .95,2005, Enhancement of single - Molecule Fluorescence Detection in Sub wave length Apertures, 117401. [8] Tineke Thio ,Pellerin K.M .,et Linke R.A.,Opt .Lett, 2001, Enhanced light transmission through a single sub wavelength apertures, 26,1972. [9] Martin-Moreno L., Garcia -Vidal F.J., Lezec H. J., et al. Rev. Lett , 2001, Theory of Extraordinary Optical Transmission through Sub wave length Hole Arrays, 86, 1114. 


\section{GEOMETRIES DES GUIDES D'ONDES POUR LE CONFINEMENT DES MODES PROPAGATIFS RESONNANTS}

\section{RESUME}

Dans ce travail, nous avons étudié le mode fondamental de 1D de deux milieu (métaldiélectrique - métal et diélectrique-métal-diélectrique), d'une structure métallique (Ag) dans la quelle est perforée une ouverture remplie d'un diélectrique. En se plaçant dans les conditions de confiner les modes résonants de surface (PPS), via la permittivité complexe de la partie métallique et celle d'un milieu diélectrique. L’indice effectif de la structure élaborée le contrôle de façon déterminante les propriétés des modes propagatifs. Pour le quel la structure jouant le rôle de guider les modes PPS.Ou le confinement suit l'ordre de grandeur de la dimension sub-longueur d'onde de la largeur w de l'ouverture perforée dans l'argent (est il est limités par la langueur d'atténuation (profondeur de peau).

Mots clés : structure métallique, indice effectif, modes PPS guidés, profondeur de peau.

\section{How to cite this article}

Aboura $\mathrm{Z}$ and Bouhafs B. Geometry of waves guides for containment of propagating resonant modes. J Fundam Appl Sci. 2011, 3(1), 68-76. 